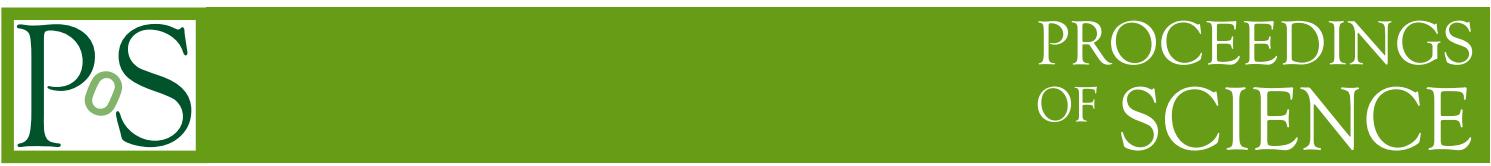

\title{
Transient neutrino emission from the Galactic Center studied by ANTARES
}

\author{
Alexis Coleiro* on behalf of the ANTARES collaboration \\ APC, Université Paris Diderot, CNRS/IN2P3, CEA/Irfu, Observatoire de Paris, Sorbonne Paris \\ Cité, 10 rue Alice Domon et Léonie Duquet, 75205 Paris Cedex 13, France. \\ E-mail: coleirodapc.univ-paris7.fr
}

\begin{abstract}
We present a search for ANTARES neutrino events in temporal coincidence with IceCube HighEnergy Starting Events (HESE), between May 2010 and December 2012. This study uses a two-point correlation function and focuses on HESE located within $45^{\circ}$ from the Galactic Center (GC). This approach is sensitive to transient emission and requires neither prior on the burst timing structure nor on the electromagnetic emission. Therefore, it provides an effective way to acquire information on the possible origin of the IceCube astrophysical signal from transient sources.
\end{abstract}

The 34th International Cosmic Ray Conference,

30 July- 6 August, 2015

The Hague, The Netherlands

${ }^{*}$ Speaker. 


\section{Introduction}

High energy neutrinos are expected to be produced in sources of cosmic rays (active galactic nuclei, X-ray binaries, supernova remnant, etc.). Since they are neutral, weakly interacting and traveling straight from their source without suffering from absorption, neutrinos are unique messengers to further understand the particle acceleration processes in such astrophysical sources.

Time integrated analyses suffer from a high background of both atmospheric muons and neutrinos. When dealing with transient emission, this background can be significantly reduced using a time-dependent approach that usually consists in searching for astrophysical neutrinos in smaller time windows around flares (see e.g. [1] and [2]). Here, we propose a model-independent approach based on the timing properties of both the ANTARES and IceCube data samples.

The IceCube collaboration announced recently ([3] , [4]) the discovery of the first extraterrestrial very-high energy neutrinos in the energy range from $30 \mathrm{TeV}$ to $2 \mathrm{PeV}$. Nine of these so-called High Energy Starting Events (HESE), occurring between June 2010 and January 2013, are positionally consistent with the Galactic Center (GC). Moreover, it was pointed out recently that two of these IceCube HESE occurred within 1 day of each other with a p-value of 1.6\% [5]. Consequently, this was interpreted as possibly the signature of a transient point source of very-high energy neutrinos in this part of the sky.

In order to search for neutrino flares in the Galactic center region, we perform a time correlation study between these nine IceCube HESE and the ANTARES dataset. This approach, requires neither prior on the burst timing structure nor on the potential electromagnetic emission. Therefore, it provides an effective way for further shedding light on the IceCube astrophysical signal possibly emitted by transient astrophysical sources. In Section 2, we first report the ANTARES and IceCube data samples, focusing on the ANTARES quality cuts optimization. Section 3 presents both the approach used in this analysis and the related discovery potential. Preliminary results are provided in Section 4.

\section{Event samples}

\subsection{ANTARES data selection}

The considered dataset was collected by the ANTARES neutrino telescope between May 01 2010 and November 30 2012. The event selection criterion has been optimized through MonteCarlo simulations to reach a constant neutrino candidate rate over time. In the following, we assume that the run-by-run variations of the ANTARES data stream are mainly due to the evolution of the data taking conditions in the sea. To accurately take into account these variations, we use the mean counting rate of the optical modules (MR) as a good measure of the real conditions in the sea. The dataset has been divided into five ranges of MR and the reconstruction quality parameter $\Lambda$ was optimized separately for each of these sub-samples to reach a constant neutrino candidate rate. Consequently, the same quality cut is used for runs subject to similar data taking conditions. The neutrino candidate rate was chosen according to a Model Discovery Potential (MDP - see [6]) optimization, varying the cut on $\Lambda$ in the sub-sample of MR related to the best data taking conditions $(50 \mathrm{kHz}<\mathrm{MR}<100 \mathrm{kHz})$. The expected signal used in the MDP optimization was defined as the last estimation of the diffuse IceCube astrophysical neutrino spectrum: $\Phi_{v}=2.06_{-0.3}^{+0.4} \times$ 
$10^{-18}\left(E_{v} / 10^{5} \mathrm{GeV}\right)^{-2.46 \pm 0.12}$ (see [7]). From the optimized $\Lambda$ cut, the corresponding neutrino candidate rate was computed. For the other ranges of MR, we computed the $\Lambda$ cut that enable to reach the previously defined neutrino candidate rate. We point out that the $\Lambda$ cut obtained for each range of MR (see Table 1) is close to the quality cut that would have been set by a separate MDP optimization in each subsample. This approach leads to a final sample consisting of 4337 events.

\begin{tabular}{|c|c|}
\hline MR range $(\mathrm{kHz})$ & $\Lambda$ cut \\
\hline $50-99$ & -5.2 \\
$100-199$ & -5.4 \\
$200-398$ & -5.5 \\
$399-794$ & -5.6 \\
$795-1585$ & -5.7 \\
\hline
\end{tabular}

Table 1: $\Lambda$ cuts for the 5 bins of MR.

\subsection{IceCube data selection}

The aim of this analysis is primary to constrain the potential transient origin of the IceCube astrophysical signal close to the GC. Among the three-year (988 days) HESE dataset, consisting of thirty-seven events (see [4]), nine of them are located within $45^{\circ}$ from the GC, of which eight occur between May 2010 and November 2012. For overlap with the considered ANTARES data sample, we will thus only consider these eight events listed in Table 2.

\section{Search methodology}

\subsection{The algorithm: Two-point correlation function}

In this analysis, we extend the two-point correlation function, which is commonly used to detect spatial clustering (see e.g. [8]) to the time domain. Thus, we consider the two-point cumulative distribution defined as:

$$
\mathscr{N}(\Delta t)=\sum_{i=1}^{N_{I C}} \sum_{j=1}^{N_{A N T}} \omega_{i j}\left[1-H\left(\Delta t_{i j}-\Delta t\right)\right]
$$

where $H$ is the Heaviside function depending on the absolute value of the temporal distance $\Delta t$. $\omega_{i j}$ are the weights assigned to each couple of IceCube (IC) and ANTARES (ANT) events, named respectively $i$ and $j$. Each weight $\omega_{i j}$ is computed according to a normal distribution centered on

\begin{tabular}{lcccccc}
\hline HESE ID & Date (MJD) & Energy $(\mathrm{TeV})$ & RA $($ Deg) & Dec $($ Deg) & Angular error (Deg) & Distance from GC (Deg) \\
\hline \hline 2 & 55351.4659661 & 117 & 282.6 & -28 & 25.4 & 14.6 \\
12 & 55739.4411232 & 104 & 296.1 & -52.8 & 9.8 & 32.5 \\
14 & 55782.5161911 & 1040 & 265.6 & -27.9 & 13.2 & 1.2 \\
15 & 55783.1854223 & 57.5 & 287.3 & -49.7 & 19.7 & 26.3 \\
22 & 55941.9757813 & 219.5 & 293.7 & -22.1 & 12.1 & 25.9 \\
24 & 55950.8474912 & 30.5 & 282.2 & -15.1 & 15.5 & 20.4 \\
25 & 55966.7422488 & 33.5 & 286.0 & -14.5 & 46.3 & 23.5 \\
33 & 56221.3424023 & 385 & 292.5 & 7.8 & 13.5 & 44.8 \\
36 & 56308.1642740 & 28.9 & 257.7 & -3.0 & 11.7 & 27.2 \\
\hline
\end{tabular}

Table 2: Properties of the IceCube HESE considered in the analysis. 
the IceCube HESE and defined as:

$$
\omega_{i j}=\exp \left(\frac{-\Delta \Omega_{i j}^{2}}{2 \sigma_{i}^{2}}\right)
$$

where $\Delta \Omega_{i j}$ is the angular distance between each couple of IceCube and ANTARES events, and $\sigma_{i}$ is the standard deviation of the $i^{\text {th }}$ IceCube HESE angular error distribution, computed from its median angular error provided in Table 2 (see also [4]). In this definition, the angular error of the ANTARES events is neglected as it is much smaller than the one of the IceCube HESE. The temporal binning $\Delta t$ is set equal to 0.01 day between 0 and 10 days and equal to 1 day between 10 and 1000 days.

\subsection{Background estimation}

To detect correlated structures in the dataset, a reference cumulative correlation distribution (considered as the background distribution) has been built by generating $10^{4}$ pseudo-experiments.

\subsubsection{Time generation}

The event times are randomized following the procedure described hereafter, assuming approximately constant neutrino candidate rates for different periods.

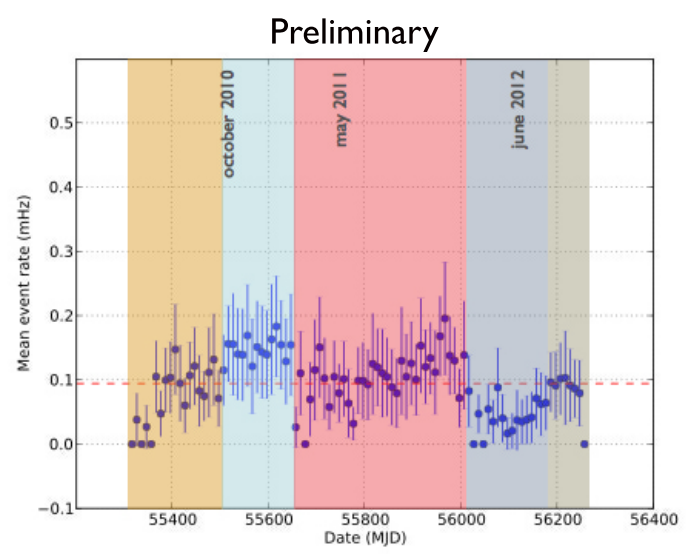

Figure 1: Mean neutrino candidate rate per bins of 10 days for the final dataset. The 5 time periods defined to generate the pseudo-experiments are indicated by the 5 colored areas.

The evolution of the mean neutrino candidate rate per bins of ten days is shown in Figure 1. From this plot, we define five sub-periods characterized by a small evolution of the mean neutrino candidate rate, which roughly follow the seasonal variation of the ANTARES data stream. For each of them, we define a mean neutrino candidate rate as:

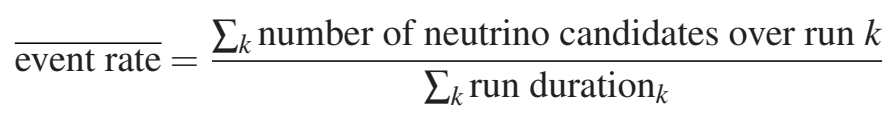

where $k$ refers to each run included in the given sub-period. 
Every sub-time period is now treated separately. For each run $k$ belonging to one given subtime period, we draw a number of neutrino candidates from a poissonian distribution of mean $\mu$ given by:

$$
\mu=\overline{\text { event rate }} \times \text { run duration }{ }_{k}
$$

where event rate is defined in Equation 3.3. Finally, we draw the event time from an uniform distribution between the run start and the run stop dates of the given run.

\subsubsection{Local coordinates generation}

The local coordinates (Azimuth; Zenith) of each event are generated from the 2D distribution of the local coordinates related to each of the five time periods defined above. Corresponding equatorial coordinates are computed from the event local coordinates, the event time and the ANTARES detector location.

This randomization process is performed $10^{4}$ times. Each pseudo-experiment is then analyzed in exactly the same way as the data to derive the normalized cumulative distribution function. All the pseudo-experiment cumulative distributions are finally averaged to compute a background estimation. Figure 2 shows the resulting two-point cumulative distribution for the background.

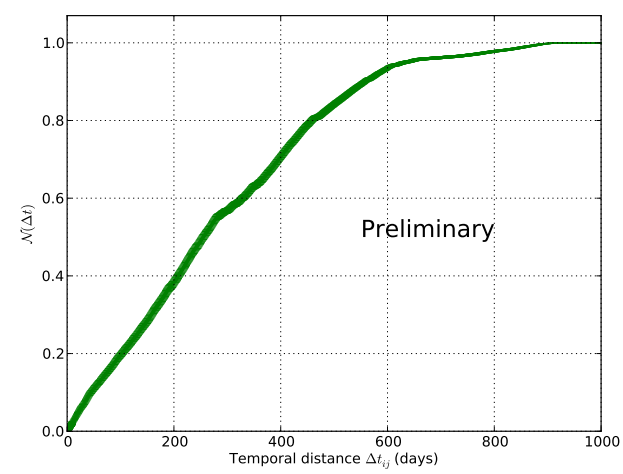

Figure 2: Cumulative two-point distribution function in the background only hypothesis (average of $10^{4}$ pseudo-experiments). The green band indicates the standard deviation of the $10^{4}$ pseudo-experiments in each bin.

\subsection{Test Statistic}

In order to detect a possible timing correlation between ANTARES events and IceCube HESE, the cumulative two-point distribution of the data is compared, bin-by-bin, with the reference distribution built according to Section 3.2. Time correlation features will thus appear as differences between these two distributions. To test the significance of such a correlation, a test statistics (TS) is defined by maximizing a value over all the timescales $\Delta t_{i}$, as given by Equation 3.5:

$$
\mathrm{TS}=\max _{\Delta t_{i}}\left[\frac{\mathrm{N}_{\mathrm{on}}-\mathrm{N}_{\mathrm{off}}}{\sigma}\right]
$$


where $\mathrm{N}_{\text {on }}$ and $\mathrm{N}_{\text {off }}$ correspond to the value of the cumulative distribution function (see equation 3.1) for a given bin $\Delta t_{i}$, computed respectively for the data and for the background. The denominator $\sigma$ is taken equal to the standard deviation of the poissonian pseudo-experiments distribution in each bin. To avoid a divergence of the test statistic (due to small $\sigma$ values related to large $\Delta t_{i}$ ) and to limit the number of trials, we scan only up to 10 days.

\subsection{Discovery potential}

In order to estimate the detection power of the analysis, we perform pseudo-experiments in which one or more event(s) is (are) replaced with one or more signal event(s) that would have come from a transient astrophysical source.

We first choose randomly one of the IceCube HESE around which the generated signal event will occur. The neutrino flare is described by a gaussian distribution of mean $\mu_{\text {flare }}$ equal to the IceCube event time and standard deviation $\sigma_{\text {flare }}$, that will be considered as an estimate of the characteristic flare duration. Thus, the generated signal event time is drawn from this probability distribution, while the angular distance between the generated signal event and the IceCube HESE, $\Delta \Omega_{i j}$, is drawn from a normal probability distribution function defined as:

$$
\operatorname{pdf}\left(\Delta \Omega_{i j}\right)=\sin \left(\Delta \Omega_{i j}\right) \times \exp \left(\frac{-\Delta \Omega_{i j}^{2}}{2 \sigma_{i}^{2}}\right)
$$

where $\sigma_{i}$ is defined as in Equation 3.2. Finally, the equatorial coordinates of the generated event are drawn randomly from all the points located $\Delta \Omega_{i j}$ degrees from the $i^{\text {th }}$ IceCube HESE. Based on the arrival time of this generated event, its equatorial coordinates are then translated into local coordinates. If these local coordinates belongs to the ANTARES visibility map (if the Zenith is $\geq \pi / 2$ ), the event is included to the generated events list. Elsewhere, it is not taken into account.

The probability for a $3 \sigma$ effect is finally provided with respect to the different combinations of flare duration and number of generated signal events (see Figure 3). We point out that the number of generated signal events corresponds to the total number of events generated around the eight IceCube HESE. Moreover, one has to keep in mind that the region of the sky around the GC is visible $\sim 70 \%$ of the time by the ANTARES telescope. This means that only $\sim 70 \%$ of the total generated signal events might be observed by ANTARES (this is particularly true for the longest flares). Thus, the effective number of ANTARES signal events per IceCube HESE is provided in the upper part of Figure 3.

\section{Results}

Figure 4 (left panel) shows the distribution of the test statistic derived for $10^{4}$ pseudo-experiments by comparing the cumulative two-point distribution of each pseudo-experiment with the cumulative reference distribution built according to Section 3.2. A test statistic value of 0.085 would correspond to a $3 \sigma$ effect. For the unblinded ANTARES dataset, a test statistic value of 0.027 is found. The probability of obtaining a TS value at least as large as this in the background only hypothesis is equal to $35 \%$, which corresponds to a $\sim 0.9 \sigma$ effect. Thus, no significant correlation has been found. The comparison between the data and the background (see Figure 4, right panel) shows that 


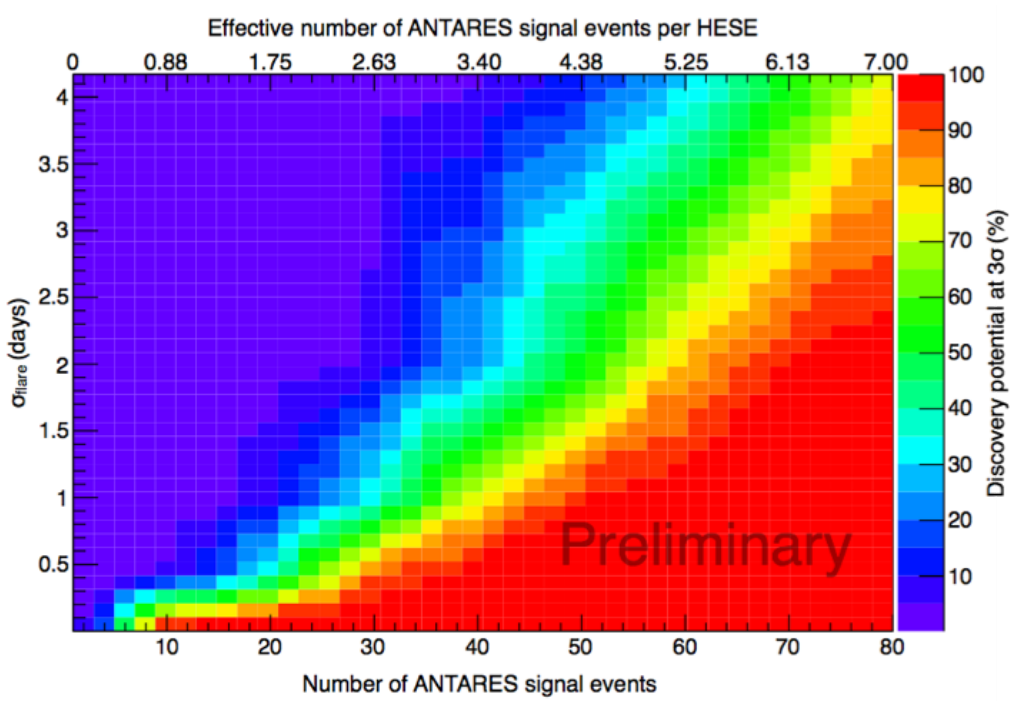

Figure 3: Discovery potential at $3 \sigma$ with respect to the flare duration $\left(\sigma_{\text {flare }}\right)$ and the total number of signal events. The effective number of signal events (see text) is also given in the upper part.

the largest deviation between the two-point cumulative distributions corresponds to a time scale of 6.1 days.

Consequently, we set upper limits on such a time correlation. Figure 5 provides the $90 \%$ confidence level upper limit on the number of ANTARES events temporally correlated with the IceCube HESE as a function of the flare duration. The blue area indicates the region excluded at a $90 \%$ confidence level. Thus, for flares shorter than $\sim 1$ hour, we can exclude the fact that at least two ANTARES events are temporally correlated with any of the 8 IceCube HESE. For larger flares, the total number of ANTARES events needed to detect a significant time correlation increases as the flare duration increases. Thus, for instance, considering a 1-day flare, one can exclude that fifteen ANTARES neutrino events arrive in correlation with any of the IceCube HESE (which corresponds roughly to 1.3 event per IceCube HESE) .

\section{Conclusion}

We have described a time-correlation analysis based on the IceCube HESE positionally consistent with the GC and the ANTARES events recorder in the period from May 2010 and November 2012 to look for the possible signature of transient neutrino emission in this region of the sky. The discovery potential was evaluated for different flare durations and numbers of signal events. No significant time correlation was found between the two samples.

\section{References}

[1] S. Adrián-Martínez et al. [ANTARES Collaboration], 2012, Astropart. Phys. 36 

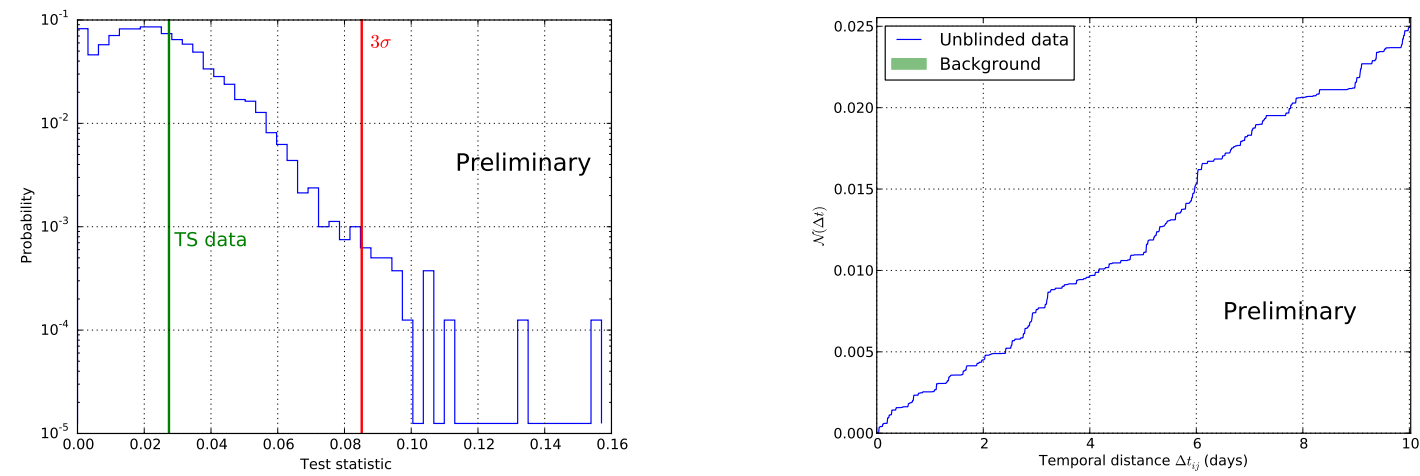

Figure 4: Left: Distribution of the test statistic (TS) in the background only hypothesis. The $3 \sigma$ probability is given by the red line while the TS value obtained for the ANTARES unblinded dataset is represented by the green vertical line. Right: Cumulative two-point distribution over 10 days. The green area corresponds to the standard deviation of each bin of the reference cumulative distribution (built according to Section 3.2), while the two-point cumulative distribution for the unblinded dataset is represented in blue.

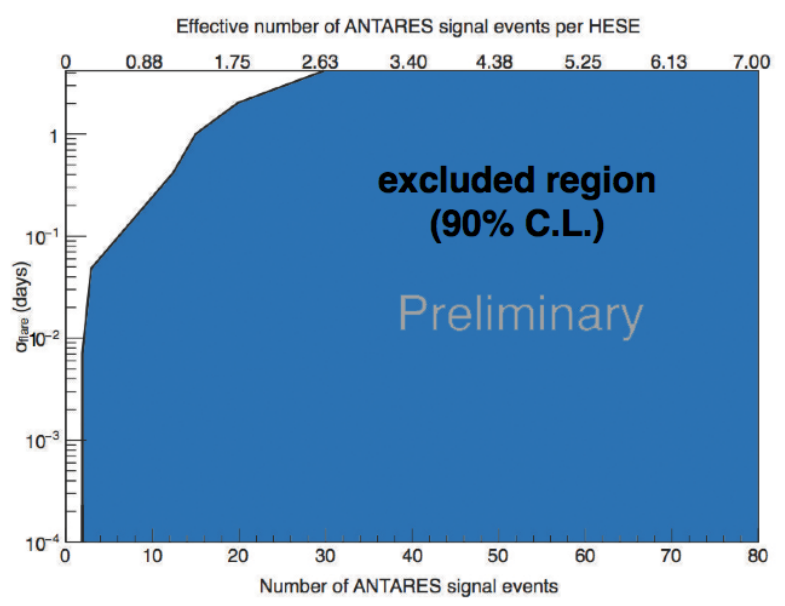

Figure 5: 90\% confidence level upper limit on the number of ANTARES events temporally correlated with the IceCube HESE as a function of the flare duration. Blue area indicates the region excluded at a $90 \%$ confidence level.

[2] S. Adrián-Martínez et al. [ANTARES Collaboration], 2014, JHEA 3-4

[3] M. G. Aartsen et al. [IceCube Collaboration], 2013, Science 342

[4] M. G. Aartsen et al. [IceCube Collaboration], 2014, Phys. Rev. Lett. 113

[5] Y. Bai et al., 2014, Phys. Rev. D 90

[6] G. C. Hill, J. Hodges, B. Hughey, A. Karle and M. Stamatikos, 2005, Proc. of PHYSTAT 2005, Oxford.

[7] M. G. Aartsen et al. [IceCube Collaboration], 2015, Phys. Rev. D 91

[8] S. Adrián-Martínez et al. [ANTARES Collaboration], 2014, JCAP 05 (2014) 001 